\title{
Combining Microscopic and Macroscopic Information for Rotation and Histogram Equalization Invariant Texture Classification
}

\author{
S. Liao, W.K. Law, and Albert C.S. Chung \\ Lo Kwee-Seong Medical Image Analysis Laboratory, \\ Department of Computer Science, \\ The Hong Kong University of Science and Technology, \\ Clear Water Bay, Kowloon, Hong Kong \\ \{liaoshu, maxlawwk, achung\}@ust.hk
}

\begin{abstract}
This paper presents a new, simple approach for rotation and histogram equalization invariant texture classification. The proposed approach is based on both microscopic and macroscopic information which can effectively capture fundamental intensity properties of image textures. The combined information is proven to be a very powerful texture feature. We extract the information at the microscopic level by using the frequency histogram of all pattern labels. At the macroscopic level, we extract the information by employing the circular Gabor filters at different center frequencies and computing the Tsallis entropy of the filter outputs. The proposed approach is robust in terms of histogram equalization since the feature is, by definition, invariant against flattening of pixel intensities. The good performance of this approach is proven by the promising experimental results obtained. We also evaluate our method based on six widely used image features. It is experimentally shown that our features exceed the performance obtained using other image features.
\end{abstract}

\section{Introduction}

Texture analysis plays an important role in computer vision and image processing. Translation, rotation, and histogram equalization invariant texture analysis methods have been of particular interest. Some researchers have considered to extract rotation-invariant features for image textures. Greenspan et al. [1] applied a set of oriented pyramid filters to an image texture and obtained a set of filtered energies. Porter and Canagarajah [2] removed the $\mathrm{HH}$ wavelet channels and combined the LH and HL wavelet channels to obtain rotation-invariant wavelet features. Haley and Manjunath 3] used Gabor filters to extract rotationinvariant features. Kashyap and Khotanzad 4 constructed an isotropic circular Gaussian Markov random field (GMRF). To capture directional information in the possibly non-isotropic textures, Deng and Clausi [5] extended the ICGMRF model 4 into anisotropic circular GMRF model. Utilizing similar circular neighborhoods, Arof and Deravi obtained rotation invariant features with 1D DFT transformation [6]. Also, Ojala et al. [7] proposed rotation invariant features 
by observing statistical distributions of uniform local binary patterns (LBP). Huang, Li and Wang extended the conventional LBP method to calculate the derivative-based local binary patterns in the application of face alignment [8].

In this paper, we propose a new approach to histogram equalization nd rotation invariant texture classification by capturing and combining both microscopic information: characteristics of local details in the textures, and macroscopic information: blob-like texture pattern in the image. The main contributions of this work are as follows. First, along the same line of Ojala et al. [7, instead of just observing the "uniform LBP", we propose to use frequency histogram of all pattern labels. At the microscopic level, it is found that this can better represent the dominant patterns in the texture images than the conventional LBP method [7]. Second, we use the Gabor filters to extract macroscopic information [10, and represent the extracted macroscopic information by computing the Tsallis entropy from the histogram of the image filtered using the circular Gabor filters because of the fact that the histogram can be transformed into a vector of generalized image entropies [9]. Finally, we found that the microscopic and macroscopic features can complement with each other effectively. This can lead to higher classification accuracy when the resolution of the image is low, e.g. $16 \times 16$, or the textures in the images are difficult to be classified. In this paper, we employ the support vector machines for performing classification, and the grid search to find the best setting of parameters which can produce the highest classification accuracy for each feature.

The performance of the proposed approach is demonstrated with three experiments on three databases: Brodatz [11, Meastex [12] and CUReT textures [13]. Excellent experimental results demonstrate that our method is able to produce, from any random rotation angle, a representation that allows for discriminating a large number of textures at other random angles. The features are computationally attractive as they can be extracted in just a few operations.

\section{Microscopic Information}

At the microscopic level, we show how to derive features based on the modified version of local binary patterns (LBP) using frequency histogram of all pattern labels in the images. It will be experimentally shown that, using frequency histogram of all pattern labels, our new features outperform the conventional LBP and other five widely used image features (see Section 4 for details). Our features are simple, and robust to image histogram equalization and rotation.

An advantage of using frequency histogram of all pattern labels over using the histogram of "uniform LBP" in the conventional LBP method [7] is that, for some kinds of textures, the dominant patterns are not mainly the "uniform LBP", especially for the textures whose edges and shapes are not regular. For example, Table 1 lists the proportions of the "uniform LBP" in some sample images obtained from the Meastex database. As listed in the table, even with different values of radius $R$, the majority of textural information cannot be effectively represented by merely considering the histogram of "uniform LBP". 
Table 1. Proportions (\%) of "uniform LBP" for some samples in the Meastex database. It shows that, for some kinds of textures, the dominant patterns are not mainly the "uniform LBP".

\begin{tabular}{|cccc|}
\hline Textures & $\mathrm{P}=8, \mathrm{R}=1 \mathrm{P}=16, \mathrm{R}=2 \mathrm{P}=24, \mathrm{R}=3$ \\
\hline Concrete0002 & 52.30 & 38.54 & 24.85 \\
Concrete0003 & 63.72 & 45.05 & 31.95 \\
Concrete0006 & 50.15 & 34.94 & 26.70 \\
Concrete0007 & 40.64 & 26.07 & 13.58 \\
Misc0000 & 58.61 & 42.50 & 30.84 \\
Misc0001 & 46.40 & 32.78 & 20.80 \\
Rock0015 & 56.80 & 41.83 & 27.32 \\
Rock0016 & 64.42 & 50.52 & 24.10 \\
Rock0017 & 44.93 & 31.70 & 16.33 \\
Rock0018 & 51.68 & 36.85 & 22.78 \\
\hline
\end{tabular}

It shows that the "uniform LBP" are not the dominant patterns in these sample images. Moreover, the approach of "uniform LBP" is not very robust against random rotation as the interpolation of pixel intensities of the rotated images can change the original "uniform" patterns into "non-uniform". Instead, the frequency histogram of all pattern labels can be more robust to random rotation as, no matter how much the images are rotated, the new patterns appear after the interpolation will also be considered in the frequency histogram of all pattern labels.

Based on the work of Ojala et. al 7], we first give the definition of pattern labels, and then describe how frequency histogram of all pattern labels is used for feature selection. Let $V$ be a vector representing the neighboring intensity values (anti-clockwise direction) at each image pixel $(x, y), V(x, y)=$ $\left(t_{0}, t_{1}, \ldots, t_{m-1}\right)^{T}$, where $(x, y)$ denote image pixel coordinates, and $t_{1}, t_{2}, \ldots$, $t_{m-1}$ represent the intensity values of $m$ equally spaced pixels around the pixel at $(x, y)$. In order to maintain rotation invariance, a circular neighborhood system is used. Therefore, $t_{1}, t_{2}, \ldots, t_{m-1}$ form a circularly symmetric neighbor set on a circle of radius $R$. Fig. 1 illustrates the circularly symmetric neighbor sets for different values of $m$ and $R$. The intensity values of the neighboring pixels are estimated using the bilinear interpolation. Let $t_{0}$ be the intensity value of a neighboring pixel, which is $(R, 0)$, to the right of the center pixel $t_{c},(0,0)$, and $t_{1}, t_{2}, \ldots, t_{m-1}$ denote the intensity values in the order of anti-clockwise from $t_{0}$. To achieve histogram equalization invariance, the intensity value $t_{c}$ at the center pixel is subtracted from the intensity values of the neighbor sets $t_{1}, t_{2}, \ldots, t_{m-1}$. A vector is defined to represent the trend of each pixel to its neighbors in the image, $\operatorname{Trend}(x, y)=\left(u\left(t_{0}-t_{c}\right), u\left(t_{1}-t_{c}\right), \ldots, u\left(t_{m-1}-\right.\right.$ $\left.\left.t_{c}\right)\right)^{T}$, where $u(x)$ is a step function, $u(x)=1$ when $x \geq 0$; else, $u(x)=0$. The vector Trend at each pixel is a highly discriminative microscopic texture feature. It is robust to histogram equalization because the sign of difference between two pixels will not be changed after performing histogram equalization. Then, a binary weighted factor $2^{i}, i=0,1, \ldots, m-1$ is assigned to 

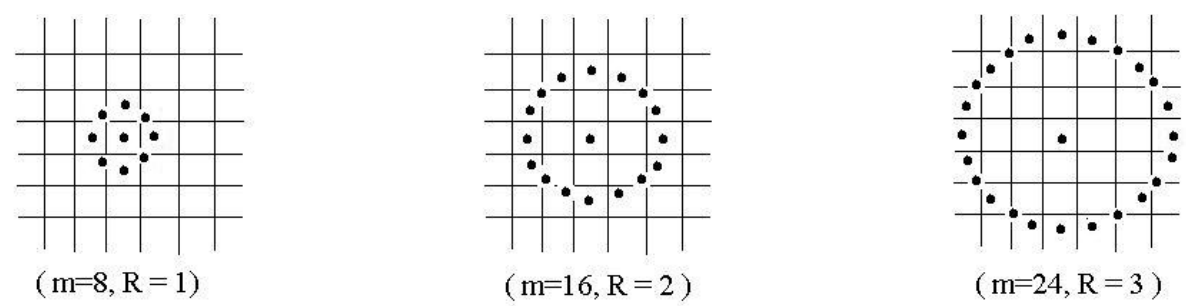

Fig. 1. Circularly symmetric neighbor sets for different values of $m$ and $R$

each element in vector Trend in order to label each pattern, which is given as,

$$
\operatorname{Mic}(m, R)=\sum_{i=0}^{m-1} u\left(t_{i}-t_{c}\right) 2^{i} .
$$

This feature is an effective representation of the information at the microscopic level because its value denotes a unique label number which describes the pattern characteristics centered at a particular pixel.

Suppose that the image is rotated by an arbitrary angle, the intensity values of $t_{i}$ will correspondingly move along the perimeter of the circle centered at $t_{c}$. Therefore, rotating the image with a particular angle naturally results in a different $\operatorname{Mic}(m, R)$ value. To remove the rotation effect and achieve rotation invariance, we need to group two pattern structures together if one can be obtained by performing rotation with the other. To assign a unique identifier to each rotation invariant group, the feature is now re-defined as,

$$
M i c^{s}(m, R)=\min (\operatorname{Cir}(\operatorname{Mic}(m, R), n)),
$$

where $n=0,1, \ldots, m-1, \operatorname{Cir}(x, n)$ performs a circular anti-clockwise bit-wise shift on the $m$-bit number by $n$ times. Unlike the conventional LBP, we consider all the pattern combinations and group them into the same rotation invariance group. Therefore, no matter how much the image is rotated (randomly rotated), we can still map each pattern in the rotated image into one group, while for the approach of local binary patterns some kinds of rotation angles will lead to rotation variant effects due to interpolation of pixels intensities of rotated images.

In the real world applications, rotation angles are not always integers or regular angles. As such, any texture classification approach should be robust to random rotation. For the approach of "uniform LBP", some patterns that are uniform will be changed to non-uniform due to the interpolation of random rotated images. To solve this problem, we propose to consider all pattern labels and use the frequency histogram to find a minimum set of pattern labels that represent $80 \%$ of the pattern labels in an image because they should correctly represent the dominant pattern labels given the image. This is more robust to random rotation because no matter how much the image is rotated, the dominant texture pattern information will be captured by the frequency histogram of 
the dominant pattern labels. According to our experimental results, it appears that the first 20 pattern labels are generally sufficient to reach or exceed $80 \%$ of the pattern labels in the image, and can effectively reflect the characteristics of the dominant textures in the image.

\section{Macroscopic Information}

Although the microscopic information can effectively represent the small, local pattern distribution in the image, it is still not sufficient to represent all the information or characteristics of the whole image. It becomes obvious especially when the resolution of the image is relatively high. To overcome the shortage of the microscopic information, we need to derive the macroscopic information in the image so that the microscopic and macroscopic information can complement well with each other. We use the circularly symmetric Gabor filters to extract macroscopic features of the image. The magnitude of the filtered image measures existence of blob-like structures in textures, which describes whether the textural pattern forms clusters. Apart from the traditional approach that averages pixel magnitude of each filtered image, we compute the Tsallis entropy 14 from the filter outputs. The reason is that the average pixel magnitude can be affected by histogram equalization and suffered by the lost of individual general information because of taking the average value. The Tsallis entropy of the filter outputs can be expressed as a linear function of the histogram and is more robust to histogram equalization.

Gabor filters are often used in texture analysis to provide features for texture classification and segmentation 15, [16. It functions over the whole image. Therefore, the features extracted from the filtered image are the macroscopic features. It is also resistant to histogram equalization as it takes the overall intensity distribution into consideration.

The traditional Gabor filter varies along one direction alone, thus making it highly orientation specific. As a result, the filter is not suitable for achieving rotation invariance. To achieve rotation invariance, we need to have circularly symmetric Gabor filter, which is given by $h(x, y)=g(x, y) e^{-2 \pi j F \sqrt{x^{2}+y^{2}}}$ where $F$ is the required center frequency. To extract macroscopic texture-based features from an image using the circularly symmetric filters, we use four circularly symmetric Gabor filters, with different center frequencies (measured in cycles/image) $F_{1}=2.0, F_{2}=3.17, F_{3}=5.04$ and $F_{4}=8.0$ so that they are spaced in geometric progression across the Fourier domain to achieve optimum coverage. These four filters overlap slightly and the Fourier domain is almost evenly covered. Finally, four filter responses to the input image can be obtained. We denote the histograms of four filter responses as $H^{1}, H^{2}, H^{3}, H^{4}$, respectively. The features at macroscopic level are then extracted from the individual histograms by computing the corresponding Tsallis entropy [14] $S_{q}=\frac{1-\sum_{j=0}^{m-1} h_{j}^{q}}{q-1}$, where $q$ is a continuous parameter, $m$ denotes the maximum bin number for the histogram, $h_{j}$ is the value of the corresponding histogram density, e.g. $H^{1}, H^{2}, H^{3}$ or $H^{4}$. The Tsallis entropies are powerful features to represent the macroscopic infor- 
mation extracted from the histogram [18. For texture classification, the four Tsallis entropies extracted from $H^{1}, H^{2}, H^{3}$ and $H^{4}$ are used as the features at the macroscopic level.

\section{Experimental Results}

We have evaluated our method on three different databases with large sets of texture images: (1) 24 source textures captured from the Brodatz album [1]; (2) all textures (28 kinds of texture in total) in the Meastex database 12, which is very challenging as most of the textures are very similar to each other; and (3) 47 textures selected from the CUReT database [13. We have also reduced the resolution of the images to study the effect of different image resolutions on classification accuracy. In the experiments, we performed histogram equalization and randomly rotated each image in order to test the robustness of our method. The robustness of our approach is compared with six commonly used image features, which are listed below.

1. Daubechies wavelet packet features (DWPF) [17] and [2]: The feature vector consisted of the $L_{2}$ norms of the images of the wavelet packet transform [17. The wavelet transform was combined with spatial sub-sampling to give critical image sampling. The rotation-invariant version DWPFRT [2] was also implemented.

2. Traditional Gabor filters (Gabor) [3]: Eight Gabor filters were chosen, spaced at the frequencies, $F=2.0,3.17,5.04$ and 8.0 and oriented at angles of 0 and 90 degrees to achieve optimal coverage in the Fourier domain. Average pixel magnitude of each filtered image was used as feature. The circularly symmetric version CGabor was also implemented.

3. Gaussian Markov random field parameters (GMRF) [19]: Each pixel was assumed to be a linear combination of the intensities of its neighboring pixels. We utilized the $4^{\text {th }}$ order neighborhood system. The linear parameters were computed with the least square estimation.

4. Anisotropic Circular Gaussian MRF parameters (ACGMRF) [5]: An improved version of the Gaussian MRF mentioned above was implemented. It was rotation invariant with strong response to directional features. In total, 36 parameters were calculated using approximated least square estimation from a $3^{\text {rd }}$ order symmetrical 24 orientation neighborhood system.

5. Multiresolution Histograms (MH) [18]: The generalized Tsallis entropy and Fisher information were computed over different resolutions. In this experiment, generalized Tsallis entropy and Fisher information were computed over three resolution levels and let the continuous parameter $q=2$.

6. Local binary patterns (LBP) [7]: The occurrence histogram of the uniform local binary patterns were computed when $P=8,16,24$ with $R=1,2,3$ respectively. The final features were the features obtained after combining the three sets of features computed over $P=8, R=1 ; P=16, R=2 ; P=24, R=3$ together. It was claimed to have the best performance of local binary patterns in Ojala et al.'s experiment [7]. 
Also, the microscopic information is denoted as Mic and the macroscopic information is denoted as Mac, and the combination of them is denoted as MicMac. We used the support vector machine (SVM) as the classifier in our experiments. SVM can perform binary classification and regression. They perform classification using the structural risk minimization principle. In particular, the SVM creates a classifier with minimized VC dimension.

\subsection{Experiments on Brodatz Database}

The image data set includes 24 texture classes from the Brodatz album [11. For each texture class, there are $25128 \times 128$ source images, in which initially we divide each $128 \times 128$ source image into 4 disjoint $64 \times 64$ subimages. As such, we have 100 samples for each texture class ( 25 source images $\times 4$ divisions). We use the first 50 samples for the training of the classifier and the other 50 images are used for the testing of the classifier.

Training and classification are first performed on the textures at their original orientation and resolution, and without histogram equalization and rotation. It produces the results listed in the first column of Table 2. Testing on the original textures only verifies the basic capability of each feature, and does not test its histogram equalization and rotation invariance. The training and testing sets are then presented after performing histogram equalization but without rotation. It yields the second column of results listed in Table 2. Then, the original training and testing sets are presented by rotating each of them in a randomly generated angle between 0 and 360 degrees (angles were uniformly distributed). It should be noted that the randomly generated angle is not necessary an integer value (e.g. generating 23.24 degree is possible), but without performing histogram equalization. The classification results are presented in the third column of Table 2. The fourth column in Table 2 gives the results of performing both histogram equalization and random rotation for each training set and testing set.

Table 2. Performance of different features of $64 \times 64,(32 \times 32)$ and [16 $\times 16]$ image resolutions in the Brodatz Database. Results of our methods are listed in the last three rows. For each test (column), the highest classification accuracy is highlighted in bold.

\begin{tabular}{|c|c|c|c|c|}
\hline \multirow{4}{*}{ Features } & \multicolumn{4}{|c|}{ Classification accuracy $\%$} \\
\hline & Original & Histogram & Randomly & Histogram Equalized \\
\hline & Textures & Equalized & Rotated & \& Random Rotated \\
\hline & & Textures & Textures & Textures \\
\hline DWPF 17 & $98.61(88.89)[80.56]$ & $89.35(72.69)[55.56]$ & $83.06(79.63)[59.72]$ & $56.94(64.81)[41.20]$ \\
\hline DWPFRT 2 & $91.67(79.17)[68.06]$ & $75.00(52.78)[43.06]$ & $91.20(78.24)[64.81]$ & $78.70(60.19)[38.43]$ \\
\hline Gabor 3 & $96.76(79.17)[56.02]$ & $91.67(62.96)[38.43]$ & $64.35(47.69)[45.37]$ & $54.63(34.26)[29.17]$ \\
\hline CGabor 3 & $90.07(62.50)[48.61]$ & $51.85(26.40)[32.90]$ & $87.87(58.80)[50.46]$ & $55.09(33.80)[29.63]$ \\
\hline GMRFs 19 & $96.70(53.40)[36.81]$ & $84.33(44.20)[27.11]$ & $44.30(24.74)[23.90]$ & $40.40(22.43)[14.62]$ \\
\hline ACGMRFs 5 & $95.22(75.00)[33.19]$ & $86.52(75.46)[42.13]$ & $93.72(76.94)[31.48]$ & $80.56(74.33)[37.96]$ \\
\hline MH 18 & $96.35(78.31)[53.68]$ & $63.61(48.74)[25.12]$ & $87.64(80.21)[56.27]$ & $54.86(41.43)[24.76]$ \\
\hline LBP 7 & $98.37(92.85)[83.24]$ & $97.44(90.07)[78.22]$ & $92.13(86.37)[80.91]$ & $91.67(84.81)[74.30]$ \\
\hline Mic & $98.61(93.96)[89.91]$ & $97.69(93.33)[83.98]$ & $94.44(89.16)[82.80]$ & $91.82(85.46)[80.93]$ \\
\hline $\mathrm{Mac}$ & $87.84(63.00)[52.12]$ & $60.73(29.25)[38.06]$ & $85.65(62.17)[55.24]$ & $63.47(39.02)[35.43]$ \\
\hline MicMac & $99.54(94.91)[93.02$ & $99.54(95.37)[92.18$ & $99.54(97.69)[92.35$ & ] $99.07(94.32)[90.11$ \\
\hline
\end{tabular}


To observe the robustness of different approaches, we reduce the original image resolution from $64 \times 64$ to $32 \times 32$, and to $16 \times 16$. To achieve the resolution reduction, we first perform histogram equalization and random rotation on the original $64 \times 64$ images. Then, we take the $32 \times 32$ and $16 \times 16$ resolution images from the $64 \times 64$ images. Table 2 , (see the numbers in brackets for $32 \times 32$ and squares for $16 \times 16$ ), correspondingly lists the results of histogram equalization and random rotation perform on the textures when all training and testing images are reduced to resolution of $32 \times 32$ and $16 \times 16$. It is observed that our method based on both microscopic and macroscopic information outperforms other six widely used image features.

\subsection{Experiments on Meastex Database}

In the Meastex database [12], images are divided into 28 kinds of textures, each image is of resolution of $512 \times 512$, and there are 69 source images. It is a very challenging database because, in 28 kinds of textures, some of the images, which are very similar to each other, are divided into two different kinds of textures. Since the Meastex database is very challenging, we do not reduce the resolution less than $64 \times 64$ pixels. To setup the experimental environment, we first divide each $(512 \times 512)$ source images into 64 disjoint $(64 \times 64)$ subimages. Half of the subimages of each texture class is used as the training sets, while the other half of the subimages of each texture class is used as the testing sets.

Table 3. Performance of different features of $64 \times 64$ image resolution in the Meastex Database. Results of our methods are listed in the last three rows. For each test (column), the highest classification accuracy is highlighted in bold.

\begin{tabular}{|c|cccc|}
\hline & \multicolumn{4}{|c|}{ Classification accuracy \% } \\
& Original & $\begin{array}{c}\text { Histogram } \\
\text { Features } \\
\text { Textures } \\
\text { Equalized } \\
\text { Textures }\end{array}$ & $\begin{array}{c}\text { Rotated } \\
\text { Textures }\end{array}$ & $\begin{array}{c}\text { Random Rotated } \\
\text { Textures }\end{array}$ \\
\hline DWPF 17 & 50.74 & 42.34 & 36.31 & 30.50 \\
DWPFRT [2] & 42.53 & 31.63 & 48.52 & 27.39 \\
Gabor [3] & 56.06 & 52.63 & 46.23 & 39.61 \\
CGabor [3] & 51.30 & 42.63 & 53.68 & 38.10 \\
GMRFs 19] & 56.78 & 32.10 & 37.52 & 24.68 \\
ACGMRFs [5] & 60.73 & 58.71 & 50.31 & 48.64 \\
MH [18] & 51.66 & 26.74 & 46.32 & 22.64 \\
LBP [7] & 58.32 & 54.75 & 57.80 & 55.94 \\
\hline Mic & 61.06 & 58.34 & 57.73 & 60.18 \\
Mac & 42.10 & 38.52 & 33.50 & 31.08 \\
MicMac & $\mathbf{8 1 . 5 7}$ & $\mathbf{8 1 . 5 7}$ & $\mathbf{8 1 . 0 6}$ & $\mathbf{8 0 . 4 8}$ \\
\hline
\end{tabular}

The experimental results are listed in Table 3, which shows that Meastex is a very challenging database because the classification accuracies are generally lower that the Brodatz database. For our approach, if we just consider the microscopic information (see bottom third row in Table 3 ), it just has a mediocre performance on the original textures $(63.40 \%)$. On the other hand, if we just consider the macroscopic information (see bottom second row in Table 3), its 
performance is not good $(42.44 \%)$ because the detailed information is lost. However, if we combine the microscopic and macroscopic information (see last row in Table 3$)$, we can see that the performance is greatly improved $(81.57 \%)$, which is better than other methods listed in the table. This is a promising performance in such challenging database. Also, even in the most difficult condition, after performing histogram equalization and random rotation, our approach can still maintain a good performance $(81.07 \%)$, its robustness against histogram equalization and random rotation is strongly implied.

\subsection{Experiments on CUReT Database}

For the CUReT database [13, there are 47 textures and each texture source image is of $320 \times 320$ pixels. We first divide each source image into 25 disjoint $(64 \times 64)$ subimages. Then, we use the first 12 subimages as the training set, and the other 13 subimages are used as the testing set. We choose the CUReT database to evaluate the performance of our approach because it contains more nature images. It is also a very challenging database. The experimental results are listed in Table 4, in which our method gives promising performance when both microscopic and macroscopic information is used.

Table 4. Performance of different features of $64 \times 64$ image resolution in the CUReT Database. Results of our methods are listed in the last three rows. For each test (column), the highest classification accuracy is highlighted in bold.

\begin{tabular}{|c|c|c|c|c|}
\hline Features & $\begin{array}{l}\text { Original } \\
\text { Textures }\end{array}$ & $\begin{array}{r}\text { Class } \\
\text { Histogram } \\
\text { Equalized } \\
\text { Textures }\end{array}$ & $\begin{array}{l}\text { fication ac } \\
\text { Randomly } \\
\text { Rotated } \\
\text { Textures }\end{array}$ & $\begin{array}{l}\text { acy } \% \\
\text { stogram Equalized \& } \\
\text { Random Rotated } \\
\text { Textures }\end{array}$ \\
\hline \begin{tabular}{l|l} 
DWPF & 17
\end{tabular} & 88.30 & 57.98 & 75.53 & 36.70 \\
\hline DWPFRT [2] & 79.26 & 35.64 & 65.96 & 32.98 \\
\hline Gabor 3 & 57.45 & 39.89 & 57.45 & 21.81 \\
\hline CGabor 3] & 45.74 & 24.47 & 48.94 & 20.56 \\
\hline GMRFs 19 & 75.85 & 53.60 & 41.52 & 38.63 \\
\hline ACGMRFs 5 & 64.89 & 63.29 & 39.36 & 38.83 \\
\hline MH 18 & 67.08 & 40.31 & 53.75 & 33.38 \\
\hline LBP 7 & 69.15 & 67.55 & 65.43 & 65.85 \\
\hline Mic & 81.38 & 77.12 & 76.60 & 77.05 \\
\hline Mac & 68.80 & 60.47 & 71.31 & 63.37 \\
\hline MicMac & 95.21 & 96.28 & 94.15 & 92.02 \\
\hline
\end{tabular}

\section{Conclusion}

It is experimentally shown that our approach is capable of effectively capturing and combining both microscopic and macroscopic information in the texture images. Moreover, its excellent classification performance in Brodatz, Meastex and CUReT databases was demonstrated experimentally. It was also shown to be robust to image histogram equalization and random rotation. Our method was compared with six widely used image features. It was shown that our approach is the most robust one. To make our method invariant to other complex textures, the macroscopic part of the method, i.e., the filters used in the method, can be modified. 


\section{References}

1. H. Greenspan, S. Belongie, R. Goodman, and P. Perona, "Rotation Invariant Texture Recognition Using a Steerable Pyramid," Proc. 12th IAPR ICPR, vol. 2, pp. 162-167, 1994.

2. R. Porter and N. Canagarajah, "Robust Rotation-Invariant Texture Classification: Wavelet, Gabor Filter and GMRF Based Schemes," IEE Proc. Conf. VISP, vol. 144, no. 3, pp. 180-188, 1997.

3. G.M. Haley and B.S. Manjunath, "Rotation-Invariant Texture Classification Using a Complete Space-Frequency Model," IEEE Trans. Img. Proc., vol. 8, no. 2, pp. 255-269, 1999.

4. R.L. Kashyap and A. Khotanzad, "A Model-Based Method for Rotation Invariant Texture Classification," IEEE Trans. PAMI, vol. 8, no. 7, pp. 472-481, 1986.

5. H. Deng and D.A. Clausi, "Gaussian MRF Rotation-Invariant Features for Image Classification," IEEE Trans. PAMI, vol. 26, no. 7, 2004.

6. H. Arof and F. Deravi, "Circular Neighborhood and 1-D DFT Features for Texture Classification and Segmentation," IEE Proc. Conf. VISP, vol. 145, no. 3, pp. 167$172,1998$.

7. T. Ojala, M. Pietikainen, and T. Maenpaa, "Multiresolution Gray-Scale and Rotation Invariant Texture Classification with Local Binary Patterns," IEEE Trans. PAMI, vol. 24, no. 7, pp. 971-987, 2002.

8. X. Huang, S.Z.Li and Y. Wang, "Shape Localization Based on Statistical Method Using Extended Local Binary Pattern," IEEE Proc. Conf. Image and Graphics, pp. $184-187,2004$.

9. J. Sporring and J. Weickert, "Information Measures in Scale-Spaces," IEEE Trans. Information Theory, vol. 45, no. 3, pp. 1051-1058, 1999.

10. I. Fogel and D. Sagi, "Gabor Filters as Texture Discriminator," Biological Cybernetics, vol. 61, pp. 103-113, 1989.

11. P. Brodatz, "Textures: A Photographic Album for Artists and Designers," New York: Dover Publications, 1966.

12. "MeasTex Image Texture Database and Test Suite," Centre for Sensor Signal and Information Processing, the University of Queensland.

13. K. Dana, B. Ginneken, S. Nayar, and J. Koenderink, "Reflectance and Texture of Real-World Surfaces," ACM Trans. Graphics, vol. 18, no. 1, pp. 1-34, 1999.

14. C. Tsallis, "Nonextensive Statistics: Theoretical, Experimental and Computational Evidences and Connections," Brazilian J. Physics, vol. 29, no. 1, 1999.

15. A.C. Bovik, M. Clark, and W.S. Geisler, "Multichannel texture analysis using localised spatial filters," IEEE Trans. PAMI, pp. 55-73, 1990.

16. A. Teuner, O. Pichler, and B.J. Hosticka, "Unsupervised texture segmentation of images using tuned matched Gabor filters," IEEE Trans. Img. Proc., pp. 863-870, 1995.

17. A. Laine and J. Fan, "Texture Classification by Wavelet Packet Signatures," IEEE Trans. PAMI, vol. 15, no. 11, 1993.

18. H. Efstathios, D. Michael, and S. Nayar, "Multiresolution Histograms and Their Use for Recognition," IEEE Trans. PAMI, vol. 26, no. 7, 2004.

19. R. Chellappa, and S. Chatterjee, "Classification of textures using Gaussian Markov random fields," IEEE Trans. ASSP, pp. 959 - 963, 1985. 